\title{
DESPERDÍCIO ALIMENTAR EM RESIDÊNCIAS NO ESTADO DO RIO DE JANEIRO: ALTERNATIVAS PARA REDUÇÃO
}

\author{
${ }^{1}$ Larissa de Oliveira Aragão \\ ${ }^{2}$ Lilian Bechara Elabras-Veiga \\ ${ }^{3}$ Simone Lorena Quitério de Souza
}

\section{RESUMO}

Em contraste com um cenário global em que a fome atinge 8,9 \% da população mundial, mais de 900 milhões de toneladas de alimentos são desperdiçados. Medidas de enfrentamento ao desperdício de alimentos estão em consonância com os Objetivos de Desenvolvimento Sustentável, estabelecidos pela Organização das Nações Unidas, na Agenda 2030. O presente estudo apresenta possíveis alternativas para redução do desperdício alimentar em residências, setor que origina a maior quantidade de desperdício. Essas alternativas foram traçadas a partir dos principais fatores que influenciam a ocorrência do desperdício, identificados através de uma pesquisa de opinião, realizada com mais de 200 famílias no estado do Rio de Janeiro, Brasil. Os resultados apontam que medidas simples e aplicáveis a todos os consumidores, tais como educação ambiental, alternativas para uma melhor gestão e aproveitamento integral dos alimentos, podem reduzir significativamente o desperdício alimentar e consequentemente contribuir para o desafio de atingir segurança alimentar, sustentabilidade ambiental e desenvolvimento socioeconômico.

Palavras-chave: Desperdício de alimentos. Objetivos de Desenvolvimento Sustentável. Segurança alimentar. Resíduos domésticos.

\section{HOUSEHOULD FOOD WASTE IN RIO DE JANEIRO STATE, BRAZIL: CIRCUMSTANCES AND ALTERNATIVES FOR REDUCTION}

\begin{abstract}
In opposite to a global scenario where chronic hunger affects 1 in every 8 or 9 people, more than 900 million tonnes of food produced is wasted every year. Food waste reduction and prevention are in accordance with the United Nations Sustainable Development Goals, 2030 Agenda for Sustainable Development. This study aims to present alternatives against households' food waste. These alternatives were drawn from the main factors that contribute to food waste, identified through a survey conducted with more than 200 families, under different socioeconomic conditions, in the state of Rio de Janeiro, Brazil. The results revealed that simple measures, that could be adopted by any household, involving environmental education, better food management alternatives, and use of unconventional parts of fruits and vegetables such as leaves, bark and seeds can reduce food waste and as a result, contribute to the challenge of achieving environmental sustainability, socioeconomic development and food security.
\end{abstract}

Key words: Food waste. Sustainable Development Goals. Food security. Household waste,

\footnotetext{
${ }^{1}$ Instituto Federal de Educação, Ciência e Tecnologia do Rio de Janeiro - IFRJ, Rio de Janeiro, (Brasil). https://orcid.org/0000-0003-2884-4208 E-mail: larissa.oaragao@gmail.com

${ }^{2}$ Instituto Federal de Educação, Ciência e Tecnologia do Rio de Janeiro - IFRJ, Rio de Janeiro, (Brasil). https://orcid.org/0000-0002-5403-018X E-mail: lilian.veiga@ifrj.edu.br

${ }^{3}$ Instituto Federal de Educação, Ciência e Tecnologia do Rio de Janeiro - IFRJ, Rio de Janeiro, (Brasil). https://orcid.org/0000-0002-8988-3465 E-mail: simone.quiterio@ifrj.edu.br
} 


\section{INTRODUÇÃO}

Em um cenário de mudanças climáticas, crescimento populacional e escassez de recursos naturais, com iminente colapso hídrico, o abastecimento alimentar nos próximos anos é de especial interesse e preocupação (Shukla et al., 2019).

No mesmo ano (2019), em que a fome atingiu 690 milhões de pessoas e outras bilhões não puderam se alimentar de maneira saudável ou nutritiva, 931 milhões de toneladas de alimentos foram desperdiçados, o que corresponde a $17 \%$ da produção mundial de alimentos. O desperdício foi medido entre alimentos vendidos para residências, varejistas, restaurantes e outros serviços alimentícios. Dentre esses, as residências representam a maior fonte de desperdício (Food and Agriculture Organization of the United Nations [FAO], 2020; United Nations Environment Programme [UNEP], 2021). O número de 690 milhões de pessoas afetadas pela fome no ano de 2019 representa um aumento de 10 milhões em relação a 2018 e de aproximadamente 60 milhões em relação aos últimos cinco anos e, como resultado da recessão econômica desencadeada pela pandemia de COVID-19, espera-se um agravamento desse cenário (UNEP, 2021).

A Agenda 2030 é um plano de ação estabelecido pela Organização das Nações Unidas (ONU), na qual dezessete objetivos subdivididos em cento e sessenta e nove metas, foram adotados por cento e noventa e três países visando alcançar o desenvolvimento sustentável em todo o mundo. Dentre estes objetivos, o Objetivo de Desenvolvimento Sustentável (ODS) 12, é referente a padrões de produção e consumo responsáveis, através de ações que estimulam a sustentabilidade em processos produtivos e na dinâmica de consumo. A meta 12.3 estabelece que metade do desperdício de alimentos per capita mundial, em níveis de varejo e consumo, deve ser reduzido até 2030. A meta estabelece ainda a necessidade de redução das perdas de alimentos ao longo das cadeias de produção e abastecimento (Assembleia Geral da ONU, 2015).

O desperdício de alimentos também está relacionado ao ODS 2: "Acabar com a fome, alcançar a segurança alimentar e melhoria da nutrição e promover a agricultura sustentável" (Assembleia Geral da ONU, 2015). A crescente demanda por alimentos, especialmente nos países em desenvolvimento, combinada às mudanças climáticas ameaça a capacidade de um suprimento alimentar global adequado (Wunderlich \& Martinez, 2018). Por isso, os recursos perdidos e os impactos produzidos em decorrência do desperdício de alimentos agravam a insustentabilidade do sistema alimentar.

Na contramão à meta 12.3 da Agenda 2030, um estudo do Boston Consulting Group (BCG) prevê que o desperdício global de alimentos aumente em mais de $30 \%$ no ano de 2030. Estima-se que 2,1 bilhões de toneladas de alimentos sejam perdidos ou desperdiçados se medidas não forem tomadas (Hegnsholt, Unnikrishnan, Pollmann-Larsen, Askelsdottir \& Gerard, 2018).

No Brasil, esse desperdício é de $114 \mathrm{~g} \mathrm{dia}^{-1}$ por pessoa, o que representa, anualmente, um desperdício de 41,6 kg de alimentos por pessoa e em média $128 \mathrm{~kg}$ por família (Porpino, Lourenço, Araújo \& Bastos 2018). No entanto, é importante ressaltar que esses valores consideram apenas refeições realizadas em domicílio, sem contabilizar o desperdício que ocorre em restaurantes, refeitórios e escolas, por exemplo, o que tornaria os resultados ainda mais alarmantes.

Quando o alimento é desperdiçado, todos os insumos utilizados para a sua produção também são perdidos. Trinta e oito por cento dos recursos energéticos consumidos pelo sistema alimentar global são utilizados para produzir alimentos perdidos ou desperdiçados e $28 \%$ das terras agricultáveis do mundo são destinadas à produção de alimentos que nunca serão consumidos (FAO, 2013).

Ao desperdício alimentar estão fortemente associados os impactos consequentes da geração de resíduos orgânicos. Com a degradação da matéria orgânica são formados gases de efeito estufa (GEE), que são responsáveis pela intensificação do aquecimento global. Acredita-se que entre 2030 e 2052 o Aquecimento Global atinja $1,5^{\circ} \mathrm{C}$ acima dos níveis pré-industriais, trazendo 
severas consequências ambientais e riscos para segurança alimentar, saúde e segurança humana (Masson-Delmotte et al., 2018).

Além disso, a degradação dos resíduos orgânicos, dispostos em aterros sanitários, ocasiona a formação de percolado ou chorume, líquido com alta carga poluidora, que se não receber o tratamento adequado, contamina o solo e a água, podendo atingir os lençóis freáticos (Azevedo et al., 2015).

O prejuízo econômico total associado ao desperdício de alimentos, considerando a valoração de custos ambientais e sociais está estimado em US\$ 2,6 trilhões por ano, o equivalente ao PIB do Reino Unido - quinta maior economia do mundo (Empresa Brasileira de Pesquisa Agropecuária [EMBRAPA], 2017; FAO 2015). Por isso, o combate ao desperdício de alimentos pode ser visto como um ponto de intersecção entre sustentabilidade ambiental, segurança alimentar e desenvolvimento socioeconômico.

Um relatório publicado pelo World Resources Institute (WRI) em 2019, projeta que, com o crescimento populacional, em 2050, será preciso aumentar em $50 \%$ a produção de alimentos para abastecer a população mundial. Os esforços para evitar uma crise de abastecimento alimentar nos próximos anos, no entanto, não devem ser direcionados apenas para incrementar a produção, mas em aproveitar melhor o que já é produzido, através da redução de perdas e desperdícios. Nesse sentido, o mesmo relatório aponta a erradicação do desperdício alimentar como indispensável para evitar uma crise de abastecimento e colapso climático (Searchinger, Waite, Hanson, Ranganathan, Dumas, Matthews \& Klirs, 2019).

No Brasil, o desperdício de alimentos está fortemente associado à cultura da fartura, tanto no momento da compra quanto na preparação de refeições (Porpino et al., 2018). Problemas associados à armazenagem e conservação dos alimentos são outros antecedentes do desperdício (Porpino, Parente \& Wansink, 2015). No entanto, o desperdício de alimentos não envolve apenas o descarte de sobras dos alimentos consumidos ou daqueles que nem chegam a ser consumidos. A tendência de desprezar partes "alternativas" dos alimentos, como folhas, cascas e talos também configura desperdício alimentar (Storck, Nunes, de Oliveira \& Basso, 2013).

Em 2018, o BCG identificou a falta de conhecimento sobre as circunstâncias para a ocorrência do desperdício de alimentos e de possíveis soluções para o problema como uma das principais justificativas para o atual cenário global de desperdício (Hegnsholt et al., 2018). No contexto brasileiro, Porpino et al. (2015) evidenciaram uma lacuna em pesquisas sobre o desperdício de alimentos em residências brasileiras e uma negativa discrepância em relação a esforços nacionais para a mitigação do desperdício alimentar entre consumidores, comparado a outros países.

Nesse contexto, um estudo realizado através de um programa de cooperação entre a União Europeia e o Brasil, liderado pela Embrapa em parceria com a Fundação Getúlio Vargas (FGVEAESP) contribuiu para um maior entendimento do comportamento de consumo e desperdício de alimentos no Brasil (Porpino et al., 2018). O relatório mapeia, contextualiza e estabelece correlações da interação dos brasileiros com o desperdício de alimentos. Além disso, quantifica o desperdício absoluto e relativo de alimentos nos lares brasileiros.

Com base no acima exposto, o objetivo desse estudo é propor alternativas para a redução do desperdício alimentar em residências a partir da identificação e discussão das principais circunstâncias envolvidas no desperdício alimentar entre famílias residentes no Estado do Rio de Janeiro (ERJ), onde 17,8 \% dos domicílios se encontravam, no ano de 2018, em situação de insegurança alimentar e nutricional e a cesta básica da capital está constantemente entre as mais caras do país, tendo atingido em dezembro de 2019 a primeira posição nacional (RIO DE JANEIRO, 2018; DIEESE, 2020). A partir da pesquisa realizada e dos resultados obtidos é possível identificar alternativas para uma melhor gestão dos alimentos e, consequentemente, para a redução do desperdício alimentar. 


\section{REFERENCIAL TEÓRICO}

Neste item serão abordados os principais impactos associados ao desperdício de alimentos, com ênfase aos impactos ambientais, cujo dimensionamento e discussão são extremamente importantes no enfrentamento ao desperdício, conforme constatado nos resultados. Em continuidade, aborda-se brevemente alguns antecedentes para o desperdício alimentar e respectivas alternativas para a redução, discutidos no item 4.

\subsection{Impactos do Desperdício alimentar}

A identificação e análise dos impactos ambientais associados ao desperdício de alimentos deve considerar toda a cadeia de suprimentos (produção, processamento, transporte, distribuição e consumo) e a disposição dos resíduos orgânicos (Hall, Guo, Dor \& Chow, 2009). Os impactos ao longo da cadeia são cumulativos e, quanto mais avançada a etapa da cadeia de suprimentos, maior a significância desses impactos. Esse aspecto é demonstrado através dos indicadores de pegada hídrica, ecológica e de carbono e ao realizar a Avaliação de Ciclo de Vida do alimento (Hoekstra, Chapagain, Aladaya, \& Mekonnen 2011; Martindale, 2017). Um alimento desperdiçado emite carbono, por exemplo, nas etapas de produção, processamento, transporte, armazenamento, distribuição, preparo pelo consumidor final e por fim, ao ser destinado a um aterro sanitário (Gráfico 1) (FAO, 2013).

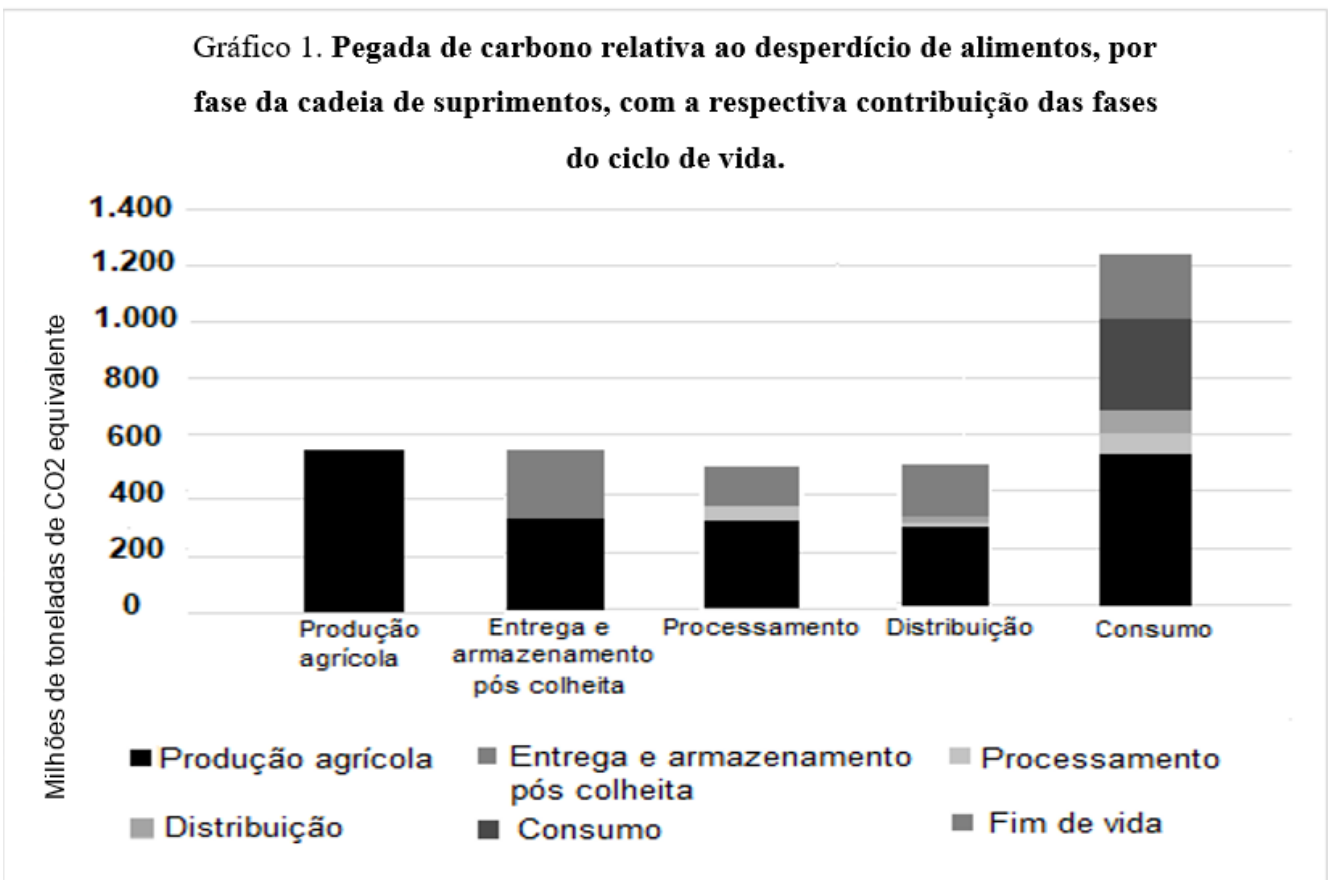

Fonte: Adaptado de Food and Agriculture Organization of the United Nations. (2013). Food wastage footprint: Impacts on natural resources - Summary report. Rome: FAO.

Segundo o World Wide Fund for Nature (WWF), o sistema alimentar é o maior consumidor de recursos naturais, o maior emissor de GEE e a principal causa de desmatamento e de perda de habitat. Além disso, é responsável pelo uso de $34 \%$ do solo e $69 \%$ da água dos corpos hídricos disponíveis (WWF, 2018).

De forma geral, o modelo atual empregado na agricultura e indústria de alimentos, no Brasil e no mundo, é predatório e prejudicial ao meio ambiente (Heck, Pilcher, Ray \& Brito, 2018). Seguindo unicamente essa lógica predominante e desconsiderando alternativas sustentáveis para a produção de alimentos, os impactos sustentam-se como um "mal necessário". No entanto, no caso 
de 931 milhões de toneladas de alimentos desperdiçados (UNEP, 2021) trata-se de um mal em vão, um impacto pelo impacto.

Quanto aos recursos hídricos, estima-se que até 2050 cerca de 5 bilhões de pessoas serão afetadas pela escassez hídrica (United Nations World Water Assessment Programme [WWAP], 2018). Por outro lado, apenas a pegada hídrica azul (consumo de água doce superficial e subterrânea) de alimentos que são produzidos e não consumidos anualmente é de cerca de $250 \mathrm{~km}^{3}$. Essa quantidade equivale à vazão anual do Rio Volga, o mais longo da Europa, e poderia suprir a demanda de todas as residências do mundo (FAO, 2013; FAO, 2016).

Ademais, outra problemática relacionada ao desperdício é a geração de resíduos orgânicos (Nahman, Lange, Oelofse, \& Godfrey, 2012). Essa questão é de especial importância, já que a partir dos processos químicos e biológicos de decomposição da matéria orgânica ocorre a formação de chorume, favorecendo a proliferação de transmissores de doenças, como insetos e roedores, e impactando a qualidade de vida da população próxima a aterros e lixões, em decorrência do odor desagradável gerado (Leal, 2016).

A degradação dos resíduos orgânicos também agrava o aquecimento global em decorrência da formação de GEE, especialmente o gás metano (CH4) e o óxido nitroso (N2O), cujos potenciais de aquecimento global são, respectivamente, 20 e 300 vezes maiores do que o do principal gás de efeito estufa, o CO2 (FAO, 2018).

A perda e o desperdício de alimentos geram de $8 \%$ a $10 \%$ de todas as emissões de GEE produzidos pelos seres humanos (Mbow et al., 2019) e a pegada de carbono dos alimentos produzidos que não são consumidos é estimada em 3.3 bilhões de toneladas de $\mathrm{CO} 2$ equivalente, sem contar as emissões pela mudança de uso do solo. (FAO, 2013).

No Brasil, onde a matéria orgânica, representa mais da metade da composição dos resíduos sólidos urbanos coletados, apenas 0,3 \% desses foi destinado para a compostagem em 2015, potencializando assim, os impactos ocasionados por esses resíduos (Zago \& Barros, 2019). A situação do Brasil nesse sentido é particularmente vulnerável, pois, segundo a Associação Brasileira das Empresas de Limpeza Pública (Abrelpe), $40 \%$ do lixo coletado no país é disposto em locais onde não existem sistemas de impermeabilização do solo e drenagem de chorume e biogás (ABRELPE, 2019).

Existe ainda uma questão fundamental atrelada ao desperdício de alimentos, cuja compreensão é determinante para dimensionar seu real impacto. Quanto mais alimento for desperdiçado, mais será necessário produzir para repor a quantidade descartada, e consequentemente, mais recursos naturais serão utilizados e mais impactos ambientais serão gerados na cadeia de suprimentos (EMBRAPA, 2017). A necessidade de produzir mais sustenta modelos de produção intensiva e acelerada que desconsideram totalmente os efeitos imediatos e de longo prazo ao meio ambiente e à saúde da população (Heck et al., 2018), bem como a crescente expansão agrícola para novas áreas e consequente perdas na biodiversidade (EMBRAPA, 2017).

Socialmente, a prática de desperdiçar alimentos aprofunda a relação de desigualdade entre a fome e o excedente de alimentos. A quantidade de alimentos desperdiçada no mundo poderia alimentar dois bilhões de pessoas, ou seja, mais que o suficiente para a erradicação da fome (ONU News, 2013). Por esse motivo, é fato que o combate ao desperdício é um importante patamar para a segurança alimentar global.

Quando o alimento é desperdiçado toda a força de trabalho e os custos diretos e indiretos necessários para obtê-lo também são desperdiçados. Os custos do desperdício de alimentos não estão associados unicamente ao valor agregado aos produtos alimentícios, mas também às externalidades produzidas, além de, no caso brasileiro representar um custo de oportunidade pelo baixo aproveitamento do valor contido nos resíduos orgânicos (Nahman et al., 2012).

Assim, o desperdício alimentar produz externalidades que serão pagas pela sociedade com recursos que poderiam ser empregados em outras áreas, como por exemplo, na educação e saúde (EMBRAPA, 2017). De fato, em 2017, o custo econômico total associado ao desperdício de alimentos no mundo estava estimado em US\$ 1 trilhão/ano. Os custos sociais e ambientais 
correspondem a US\$ 900 bilhões e US\$ 700 bilhões respectivamente (FAO, 2015). Apesar das tentativas de valoração, os custos sociais e ambientais são imensuráveis.

Assim, para minimizar as perdas associadas ao desperdício de alimentos, cidades europeias vêm sendo pioneiras na adoção de um modelo de Economia Circular (EC) de alimentos (Comissão Europeia [CE], 2015).

No Brasil, onde a matéria orgânica, representa mais da metade da composição dos resíduos sólidos urbanos coletados, apenas $0,3 \%$ desses foi destinado para a reciclagem (compostagem) em 2015, potencializando assim, os impactos ocasionados por esses resíduos (Zago \& Barros, 2019). A situação do Brasil nesse sentido é particularmente vulnerável, pois segundo a Associação Brasileira das Empresas de Limpeza Pública (Abrelpe), $40 \%$ do lixo coletado no país é disposto em locais onde não existem sistemas de impermeabilização do solo e drenagem de chorume e biogás (ABRELPE, 2019).

\subsection{Desperdício de alimentos: antecedentes e alternativas}

As principais causas atribuídas ao desperdício de alimentos são em maior parte evitáveis (Quested \& Murphy, 2014). Cartazes de uma campanha Norte-americana na II Guerra Mundial documentam uma preocupação antiga em relação ao desperdício alimentar (Baptista, Campos, Pires \& Vaz, 2012). Notavelmente, medidas indicadas nesta campanha para prevenir o desperdício, como "comprar com sabedoria e comer tudo", estão relacionadas com as que hoje ainda são apontadas como as principais causas para o desperdício alimentar: compras abundantes e sem planejamento, que favorecem que os produtos expirem, não sendo utilizados no prazo de validade; cozinhar e servir alimentos em excesso (Quested \& Murphy, 2014).

É impossível dissociar a dinâmica de consumos de alimentos do contexto social e sobretudo cultural (Porpino, 2018). Nesse sentido, Stangherlin e Barcellos (2018) destacam a influência de fatores externos, pessoais e comportamentais nos hábitos de consumo e na relação com o desperdício alimentar.

Observa-se na cultura latina uma tendência para fartura. A preferência pela abundância começa na etapa de aquisição de produtos alimentícios e pode ser um fator intensificador do desperdício. (Porpino, 2018). O hábito de fartura também é observado no momento de preparo dos alimentos e é motivado por simbolismos sociais e culturais como hospitalidade (Porpino, Parente \& Wansink, 2015). Combinada à significativa predileção por "comida fresca" (preparada recentemente) entre os brasileiros, a fartura à mesa intensifica o desperdício pois as sobras das fartas refeições muitas vezes são descartadas por serem encaradas como "comida dormida" (Porpino et al., 2018). Em geral, a resistência ao consumo de alimentos que não são recém preparados é apenas uma preferência pessoal, mas para algumas pessoas está associada à preocupação com a segurança dos alimentos (Porpino, 2018).

Por fim, algumas pessoas armazenam as sobras com a intenção de consumi-las mais tarde e ainda outras fariam isso apenas para postergar a sensação de culpa ao jogar alimentos fora (Evans e Welch, 2015; Porpino et al., 2015). No entanto, mesmo entre os que têm intenção de consumo, os alimentos podem acabar esquecidos na geladeira e, por fim, não estar mais próprios para o consumo (Evans \& Welch, 2015). A esse respeito Baptista et al., (2012) sinaliza que os refrigeradores e o congelador poderiam assumir um "papel duplo" pois ajudam a preservar os alimentos, mas, por outro lado, podem se tornar "depósitos onde os produtos ficam esquecidos, antes de serem encaminhados para o lixo" (Baptista et al., 2012, p. 47).

Ainda associado ao armazenamento dos alimentos, observa-se que a conservação inadequada ocasiona a perda de qualidade ou deterioração dos alimentos e, por fim, ao seu descarte (Tortora, Case \& Funke, 2016; Stangherlin \& Barcellos 2018). Nesse sentido, o armazenamento inadequado entre os consumidores está muitas vezes associado à falta de conhecimento. Além disso, algumas instruções sobre armazenamento podem ser conflitantes e deixar os consumidores ainda mais confusos (Hebrok \& Boks, 2017).

\begin{tabular}{|l|l|l|l|l|l|}
\hline Rev. Gest. Soc. Ambient. & São Paulo (SP) & v.15 & p.01-18 & e02739 & 2021. \\
\hline
\end{tabular} 
Dessa forma, medidas associadas ao planejamento adequado das compras e do preparo de refeições e conhecimento de hábitos corretos de armazenamento demonstram-se importantes aliados contra o desperdício alimentar em sua principal origem: as residências (FAO, 2019; UNEP, 2021). Além disso, o aproveitamento integral de alimentos reduz o desperdício e agrega valor nutritivo à alimentação.

Em uma convergência entre gastronomia, nutrição, economia e sustentabilidade, o aproveitamento integral de alimentos, permite reduzir a quantidade de resíduos orgânicos destinados ao lixo, suprir necessidades nutricionais da população, incrementar a dieta alimentar e combater os impactos ambientais e econômicos do desperdício (Laurindo \& Ribeiro, 2014).

Partes comumente não utilizadas no preparo de alimentos como cascas, talos, sementes ou folhas podem ser ricas em nutrientes essenciais para o bom funcionamento do organismo humano, por exemplo: carboidratos, proteínas, minerais e fibras, além de outras substâncias benéficas como polifenóis, importante agente antioxidante (Storck et al., 2013). Um estudo realizado por Storck et al. (2013) avaliou a composição centesimal e a aceitabilidade de 13 receitas utilizando folhas, talos, cascas e sementes de vegetais. $\mathrm{O}$ valor de proteínas encontrado nas partes descartadas de todos os alimentos foi superior ao encontrado nas partes habitualmente consumidas. O mesmo acontece no caso de minerais, quantificados através do teor de cinzas. As fibras e polifenóis também foram encontrados em maior parcela nas partes não convencionais, na maioria dos alimentos analisados.

As amostras analisadas em folhas, talos e na maioria das cascas, com exceção das sementes, apresentaram baixo valor calórico, inferior ao das partes habitualmente consumidas, ou seja, é possível obter excelentes índices nutritivos sem grande impacto na dieta calórica (Storck et al., 2013). Essa característica é mais um ponto a favor do aproveitamento integral de alimentos, em um país onde mais da metade da população tem excesso de peso e os índices de obesidade crescem (Dias \& Verona,2019).

\section{MÉTODO}

A metodologia aplicada neste estudo foi pesquisa exploratória, descritiva de procedimento bibliográfico, de abordagem qualitativa e semiquantitativa. O estudo foi realizado a partir da adaptação da metodologia aplicada ao Relatório Final de Desperdício de Alimentos (Diálogos Setoriais União Europeia - Brasil) (Porpino et al., 2018).

No estudo citado, adotou-se uma metodologia segmentada em fases qualitativa e quantitativa. A fase qualitativa inicial seguiu-se através da observação de consumidores em feiras livres e supermercados na cidade de São Paulo e da condução de entrevistas para investigar comportamentos de compra, hábitos de consumo e gestão de alimentos e percepções acerca do desperdício alimentar. Na fase quantitativa foi aplicado um questionário sobre hábitos de consumo baseado na metodologia desenvolvida por van Geffen, van Herpen e van Trijp, (2017), devidamente adaptada às especificidades brasileiras e aos resultados da fase anterior. O questionário abordou hábitos de consumo e desperdício de alimentos. Um pequeno grupo participou ainda de um diário alimentar, documentado através de fotos de seus hábitos alimentares. A chamada "fase qualitativa final" seguiu-se a partir de análise do conteúdo de mídias digitais (big data), com o objetivo de analisar quais as principais relações estabelecidas com o tema Desperdício de Alimentos pelos brasileiros e qual o público mais engajado com a questão (Porpino et al., 2018).

Para o presente estudo, os métodos e análises foram adaptados a um menor grupo, restrito ao estado do Rio de Janeiro, enquanto o estudo de Porpino et al., (2018) foi conduzido entre famílias em todo o território brasileiro. Além disso, no presente estudo não foram utilizados testes estatísticos e análise por coeficientes de correlações.

Cumpre ressaltar que para este trabalho foi utilizada amostra por conveniência (Guimarães, 2018) não tendo ocorrido, portanto, seleção de indivíduos/domicílios por meio de amostragem probabilística. Por esse motivo, os resultados encontrados estão circunscritos ao grupo de respondentes, não tendo este artigo a pretensão de realizar inferências estatísticas. 
Este estudo foi conduzido em três etapas: Primeiro, pela análise das percepções de diferentes indivíduos acerca do tema desperdício alimentar em residências e de suas principais causas, através de conversas informais com moradores do ERJ. Em seguida, foi conduzida uma Pesquisa de opinião online através da Plataforma Google Forms, que se mostrou eficiente para fins similares de pesquisa em estudos conduzidos em países europeus para analisar o desperdício alimentar em residências (Bravi, Francioni, Murmura, \& Savelli, 2020).

As questões foram estruturadas a partir da observação na primeira etapa, nas quais foram abordados os seguintes aspectos: hábitos de consumo, comportamentos ou contextos que influenciam na gestão dos alimentos, planejamento das refeições, armazenagem e preparo de alimentos, principais causas do desperdício e gêneros alimentícios envolvidos, aproveitamento integral de alimentos, percepção e colocação dos respondentes diante do desperdício e quais práticas já realizam ou acreditam ser importantes para combatê-lo. Os respondentes foram orientados a representar a percepção de toda a família diante das questões apresentadas.

A pesquisa foi divulgada em mídias sociais entre grupos aleatórios de indivíduos. Três questões de resposta "sim" e "não" foram utilizadas como critério para filtrar e coletar respostas somente de participantes que se enquadravam à proposta da pesquisa. A primeira questão-filtro garantiu a coleta de apenas uma resposta por família. A segunda questão-filtro teve a finalidade de limitar a abrangência de estudo de modo que não fossem coletadas respostas de famílias não residentes do ERJ. Por fim, foi preservado o critério adotado no Relatório Final de Desperdício de Alimentos (Porpino et al., 2018), de que os respondentes escolhidos fossem responsáveis pela compra e preparo da maior parte de alimentos que consomem, uma vez que o objeto de estudo é o desperdício em residências. Dessa forma, a terceira e última questão-filtro selecionava apenas respondentes cuja família comprasse ou preparasse mais da metade dos alimentos consumidos em uma semana.

A última etapa consistiu no tratamento das respostas coletadas e discussão dos resultados obtidos, a partir dos quais são propostas alternativas para prevenção dos principais fatores associados ao desperdício alimentar. Enquanto estudo qualitativo, o procedimento analítico utilizado refere-se à interpretação das respostas coletadas, observando critérios de confiabilidade destacados por Paiva, Leão e Mello (2011, p. 201): “Assim, é tarefa do pesquisador realizar uma análise objetiva dos fatos sociais, independentemente da atividade humana ser de caráter subjetivo, logo, a descrição rica e detalhada será critério tanto de confiabilidade quanto de validade." A discussão e apresentação de alternativas é realizada através de levantamento bibliográfico. Foi utilizado, para levantamento, o buscador Google Scholar, possuindo como critério de exclusão artigos publicados antes de 2010 .

\section{RESULTADOS E DISCUSSÕES}

A pesquisa de opinião realizada obteve duzentas respostas completas, tendo alcançado famílias de todas as mesorregiões geográficas do estado do Rio de Janeiro (IBGE, 2019), com destaque para a mesorregião metropolitana, que concentrou a maior quantidade de respostas, mais especificamente a cidade do Rio de Janeiro e a Baixada Fluminense.

Os resultados da pesquisa são apresentados e discutidos a seguir.

\subsection{Frequência e causas do desperdício}

A pesquisa apontou que 46,0 \% das famílias desperdiçam alimentos frequentemente em sua residência, 7,5\% desperdiçam com muita frequência, 46,0 \% relatam que o desperdício ocorre raramente. Apenas uma família $(0,5 \%)$ afirmou nunca desperdiçar alimentos e por isso representam $100 \%$ da composição dessa frequência de desperdício nas posteriores análises que consideram essa variável. É possível que a tendência dos indivíduos em minimizar o desperdício que ocorre em suas 
próprias residências (Neff, Spiker e Truant, 2015; Porpino et al., 2018) tenha interferido nos resultados do presente estudo, uma vez que conta apenas com a auto avaliação das famílias.

Das razões apontadas como principal causa para desperdício de alimentos obteve-se (Tabela 1): sobras e alimentos armazenados por um longo período (40,7 \%); alimento estragado (34,2 \%); servir-se de porções maiores do que será consumido (12,6\%); alimento fora da validade $(6,5 \%)$ e evitar guardar porções de uma refeição para outra $(5,5 \%)$. O motivo dos alimentos estragarem foi atribuído a problemas com armazenamento antes e após o consumo (21,0\%), esquecimento (51,5 $\%)$ e quantidade excessiva de alimento preparado $(27,5 \%)$.

Tabela 1- Principais motivos para ocorrência de desperdício

\begin{tabular}{|c|c|}
\hline Motivo para ocorrência de desperdício & \% \\
\hline Sobras e alimentos armazenados por um longo período & 40,7 \\
Alimento estragado & 34,2 \\
Servir-se de porções maiores do que o consumido & 12,6 \\
Alimento fora da validade & 6,5 \\
Evitar guardar e/ou consumir alimentos de uma refeição para outra & 5,5 \\
\hline Total & 100 \\
\hline
\end{tabular}

Fonte: Autores, 2020

Com respeito ao grupo dos alimentos mais desperdiçados, a maior parte $(49,7 \%)$ é composta por cereais e grãos, o que pode ser explicado pela abundância desse grupo na alimentação brasileira, no tradicional prato arroz e feijão. Hortaliças representam 31,2 \%; frutas 13,1 \%, leites e derivados $4,0 \%$ e carnes $2,0 \%$.

\subsection{Percepção sobre o desperdício}

Quanto à percepção sobre o desperdício de alimentos, 79,0\% dos respondentes afirmam já ter pensado sobre o desperdício de alimentos que ocorre em sua residência e que isso os incomodara, $8,5 \%$ já pensaram sobre a ocorrência do desperdício em suas residências e, no entanto, não se sentiram incomodados e $12,5 \%$ nunca pararam para pensar sobre o assunto.

A diferença entre a quantidade de pessoas que se sentem incomodadas com o desperdício que ocorre em suas residências em relação às que afirmam desperdiçar alimentos "frequente" ou "muito frequentemente" pode ser mais um indicador de que talvez os resultados de frequência do desperdício sejam subestimados.

A principal motivação para evitar o desperdício apontada notavelmente por $46 \%$ dos respondentes são os impactos econômicos que isso representa, principalmente no âmbito familiar, seguida por questões morais como parecer errado jogar alimentos no lixo enquanto outros passam fome, e por último, questões ambientais (Tabela 2).

Tabela 2. Motivação para evitar o desperdício de alimentos

\begin{tabular}{|c|c|}
\hline Motivação & \% \\
\hline Questões econômicas & 46,0 \\
Questões morais & 31,0 \\
Questões ambientais & 23,0 \\
\hline Total & 100 \\
\hline
\end{tabular}

Fonte: Autores, 2020

Similarmente, em estudo conduzido por Neff et al., (2015) a principal motivação apontada pelos participantes da pesquisa para reduzir o descarte de alimentos foi econômica, seguida por preocupação com questões sociais e, somente em último lugar, preocupação com questões ambientais. Ainda, em uma escala de importância, 22\% dos participantes do estudo afirmaram que 
preocupações ambientais não tinham importância alguma como motivação para redução do desperdício (Neff et al., 2015).

\subsection{Consciência ambiental e desperdício de alimentos}

Com relação à consciência ambiental, numa escala de um a cinco referente ao nível de preocupação com questões ambientais, a maioria dos respondentes $(47,5 \%)$ declarou que sua família demonstra-se preocupada; $31 \%$ estariam muito preocupadas e 4,0 \% estariam até mesmo engajadas com questões ambientais. Por outro lado, 13,5 \% dos respondentes declararam indiferença sobre questões ambientais e 4,5 \% afirmaram não ter nenhum tipo de preocupação com essas questões.

Buscou-se estabelecer uma correlação entre a frequência do desperdício e o grau de consciência ambiental declarado pelas famílias. No gráfico 2 é possível observar que, a diminuição da frequência do desperdício está associada a um notável aumento da concentração de famílias com grau de interesse ambiental entre 4 e 5 (muito preocupadas e engajadas com questões ambientais, respectivamente). Enquanto isso, à medida que a frequência do desperdício aumenta, a concentração de famílias com maior grau de interesse ambiental diminui. Ou seja, famílias com maior consciência ambiental tendem a desperdiçar menos alimentos.

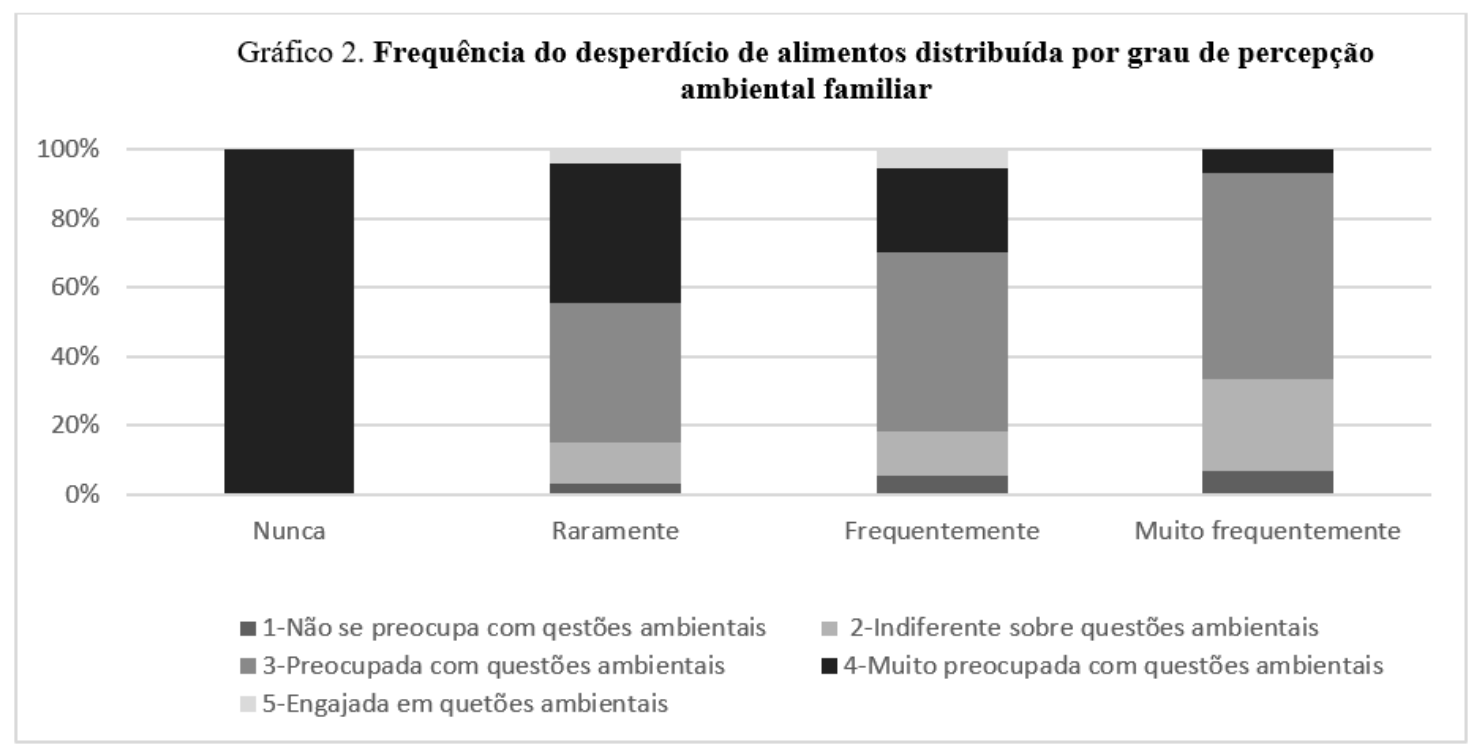

Fonte: Autores, 2020

\subsection{Planejamento}

Nas etapas de aquisição de alimentos, preparo e ao servir-se, o planejamento é um fator determinante para evitar ou intensificar o desperdício. O planejamento adequado evita a ocorrência de sobras, que muitas vezes são descartadas, esquecidas ou acabam por estragar. Além disso, o mau planejamento no momento da aquisição contribui para que alguns produtos pereçam ou ultrapassem o prazo de validade. Por isso, antes de comprar alimentos é importante seguir dois passos: saber o que existe na residência e fazer uma lista de compras adequada a essa observação e ao plano de refeições da família (FAO, 2019; Stangherlin \& Barcellos, 2018). Por fim, deve-se comprar conforme o planejado. A não utilização de listas pode favorecer compras por impulso, que mais tarde podem ser desperdiçadas. Aplicativos de celular facilitam a criação de listas de compras virtuais e personalizadas, além de permitirem visualizar um inventário das últimas compras realizadas. 
Nesse sentido, 87,0 \% das famílias declararam fazer algum tipo de planejamento antes de ir às compras, no entanto, mais de $1 / 3$ dos que realizam planejamento não chegam a segui-lo, comprando mais do que o necessário.

No momento da compra é importante ter atenção aos rótulos para analisar se o produto poderá ser consumido dentro do prazo de validade estipulado e, ao organizar os produtos comprados e planejar as refeições, adotar o sistema PVPS (Primeiro que vence, primeiro que sai) (Vasques \& Madrona, 2016). Nesse sistema, os alimentos com validade mais próxima ao vencimento são posicionados à frente dos que possuem validade mais longa, para que sejam utilizados primeiro.

Por fim, ao planejar o preparo de uma refeição deve-se levar em conta quais produtos já estão em uso e o que já está na geladeira para aproveitar sobras. Quanto a não consumir todo o alimento de que se serviu, estudos apontam a redução de resto-ingestão após realização de trabalhos voltados para a conscientização contra o desperdício de alimentos (Borges, Souza, Pinho, \& Pinho, 2019; Viana \& De Souza, 2016).

\subsection{Armazenamento e conservação dos alimentos}

A correta conservação e as condições de armazenamento, antes e após o preparo, são essenciais para reduzir a perda de alimentos por alteração de suas propriedades, principalmente as de origem microbiana, bem como para manter a condição de segurança alimentar. Problemas com armazenagem foram apontados por cerca de $21 \%$ dos respondentes como principal causa de ocorrência de alimento estragado (um antecedente do desperdício de alimentos).

Os métodos de conservação alimentar são baseados em técnicas que evitam ou retardam alterações de origem microbiana, enzimática, química e física nos alimentos, aumentando assim sua vida útil (Vasconcelos \& Melo Filho, 2010). As principais alterações a serem evitadas são as de origem microbiana, devido ao risco que podem apresentar para a saúde humana. Os principais microrganismos deteriorantes e patogênicos encontrados nos alimentos apresentam temperatura ótima de crescimento à temperatura ambiente (mesófilos), entre $25{ }^{\circ} \mathrm{C}$ e $40{ }^{\circ} \mathrm{C}$, portanto temperaturas mais baixas reduzem sua velocidade de reprodução, mantendo os alimentos adequados ao consumo por mais tempo (Tortora et al., 2016). A conservação pelo frio também desfavorece a ocorrência de reações químicas e enzimáticas indesejáveis (Vasconcelos \& Melo Filho, 2010). O resfriamento é indicado para situações em que o alimento será consumido em menor tempo e o congelamento, por sua vez, para alimentos que se deseja conservar por um maior período.

Algumas iniciativas divulgam orientações sobre o armazenamento adequado para grupos alimentares e alimentos que demandam cuidados específicos no momento de acondicionamento e conservação. Por exemplo, através do Programa da Embrapa Hortaliças: "Hortaliça não é só salada" é possível encontrar instruções específica sobre como acondicionar e conservar cada uma das 50 hortaliças mais consumidas no Brasil.

Independentemente da forma de conservação dos alimentos, quando forem utilizados recipientes para o armazenamento, estes devem ser preferencialmente transparentes, para facilitar a visualização, reduzindo a perda por esquecimento, apontada por mais da metade das famílias como principal causa para ocorrência de alimento estragado. Além disso, é importante que os recipientes apresentem boa vedação, pois uma maior disponibilidade de oxigênio torna o meio favorável para o crescimento de microrganismos aeróbios, como bolores e, portanto, diminui a vida útil dos alimentos (Nespolo, de Oliveira, Pinto \& Olivera, 2015). Cuidados na manipulação também são essenciais para manter os alimentos sadios, como correta higienização do manipulador e do ambiente (Boaventura, Frades, Weber \& Pinto, 2017). 


\subsection{Aproveitamento integral de alimentos}

Apenas 23,5 \% dos respondentes afirmam nunca utilizar partes não convencionais dos alimentos como cascas, sementes e talos, $37,5 \%$ utilizam raramente. Outros 24,5\% declaram fazer uso regular das partes alternativas e uma minoria de $14,5 \%$ afirmam utilizá-las com muita frequência.

O principal fator limitante para o uso integral dos alimentos é o desconhecimento de receitas, identificada por $76,5 \%$ dos respondentes como o motivo de não utilizar partes não convencionais dos alimentos com maior frequência (Tabela 3).

Iniciativas promovidas por diferentes instituições, programas e sites de culinária divulgam receitas para o aproveitamento integral de alimentos. Por exemplo, o MESA BRASIL SESC RJ, em 2018 publicou uma seleção de receitas intitulada "Pensando dentro e fora da casca", com receitas de doces, salgados e bebidas para utilização integral dos alimentos.

O segundo principal motivo apontado por $13,5 \%$ dos respondentes como limitante para o aproveitamento integral de alimentos foi a visão de que a prática é desnecessária. Ainda 8,0\% dos respondentes acreditam que os preparos não teriam um bom paladar e uma minoria de 2,0 \% acredita que partes alternativas dos alimentos não teriam valor nutricional (Tabela 3).

Tabela 3. Fatores limitantes para o aproveitamento integral de alimentos

\begin{tabular}{|c|c|}
\hline Fator limitante para aproveitamento integral de alimentos & $\%$ \\
\hline Desconhecimento de receitas & 76,5 \\
Ver a prática como desnecessária & 13,5 \\
Crença de que os preparos teriam gosto ruim & 8,0 \\
Crença de que os preparos não teriam valor nutricional & 2,0 \\
\hline Total & 100 \\
\hline
\end{tabular}

Fonte: Autores, 2020

Em análise sensorial de 13 receitas utilizando partes não convencionais dos alimentos, Storck et al. (2013) relataram que $77 \%$ das preparações obtiveram notas acima de 5, numa escala de 1 a 7 , na qual as notas acima de 5 significavam percepções entre "gostei" e "gostei muitíssimo", além de demonstrarem excelentes índices nutritivos. Outros estudos também atestam excelentes índices de aceitabilidade para pratos preparados à base de partes não convencionais dos alimentos (Aiolfi \& Basso, 2013, Bressiani, Schwarz, Gatti, Demário \& Freire, 2017).

Portanto, o uso integral de alimentos em maior escala depende de um esforço de sensibilização e familiarização com a prática, para entendimento de sua importância e necessidade, valor nutricional e boa aceitabilidade dos preparos.

\subsection{Desafios e oportunidades}

A partir dos principais fatores associados ao desperdício alimentar em domicílio observados através deste estudo, foi possível identificar oportunidades para ação de modo a mitigar as principais causas de desperdício, onde cada desafio é uma oportunidade para prevenção (Quadro 1). 
Quadro 1. Desafios e Oportunidades

\begin{tabular}{|c|c|}
\hline $\begin{array}{l}\text { Principais motivos para ocorrência de } \\
\text { desperdício (Desafios) }\end{array}$ & Oportunidades para redução do desperdício \\
\hline \multirow{2}{*}{$\begin{array}{l}\text { Sobras e alimentos armazenados por um longo } \\
\text { período }\end{array}$} & Planejamento ao comprar e preparar refeições \\
\hline & $\begin{array}{l}\text { Reutilização ou aproveitamento em novos preparos } \\
\text { (Porpino et al., 2018) }\end{array}$ \\
\hline \multirow{2}{*}{ Servir-se de porções maiores do que o consumido } & $\begin{array}{l}\text { Educação ambiental (Borges et al., 2019; Viana \& De } \\
\text { Souza, 2016). }\end{array}$ \\
\hline & Planejamento ao servir-se \\
\hline \multirow{2}{*}{$\begin{array}{l}\text { Evitar guardar e/ou consumir alimentos de uma } \\
\text { refeição para outra }\end{array}$} & Planejamento ao preparar refeições \\
\hline & Educação ambiental \\
\hline \multirow[t]{2}{*}{ Alimento que passou da validade } & $\begin{array}{l}\text { Planejamento ao comprar: listas de compras, } \\
\text { aplicativos (FAO, 2019; Stangherlin \& Barcellos, } \\
\text { 2018) }\end{array}$ \\
\hline & Método PVPS (Vasques \& Madrona, 2016) \\
\hline \multirow{2}{*}{ Alimento estragado } & $\begin{array}{l}\text { Hábitos corretos de armazenamento, conservação e } \\
\text { manipulação: refrigeraçãa e congelamento, } \\
\text { conhecimento das especificidades de cada produto, } \\
\text { recipientes apropriados (Tortora et al., 2016). }\end{array}$ \\
\hline & $\begin{array}{l}\text { Planejamento ao comprar e preparar: tempo de } \\
\text { consumo (Stangherlin \& Barcellos, 2018) }\end{array}$ \\
\hline Descarte de partes alternativas & $\begin{array}{l}\text { Aproveitamento integral de alimentos (Storck et al., } \\
\text { 2013; Laurindo \& Ribeiro, 2014) } \\
\text { Efetividade em divulgação de receitas, educação } \\
\text { ambiental, familiarização com a prática }\end{array}$ \\
\hline
\end{tabular}

Fonte: Autores, 2020

\subsection{Opinião dos respondentes sobre como reduzir o desperdício de alimentos}

$\mathrm{Na}$ única questão discursiva da pesquisa de opinião, cuja resposta era opcional, os participantes foram convidados a relatar quais estratégias acreditavam ser mais eficazes no combate ao desperdício de alimentos. Também foram consideradas as respostas mais recorrentes observadas durante conversas informais conduzidas na etapa que antecedeu a elaboração da pesquisa de opinião. Nos dois casos, observou-se que a maior parte dos respondentes levou em conta experiências pessoais para formular suas respostas.

A maior parte das respostas se referia ao planejamento para compra, preparo de alimentos e ao servir-se, seguida pela alternativa de redução de consumo. Também foram apontadas alternativas relacionadas à conservação e manuseio dos alimentos e até mesmo referentes a "pensar no próximo".

Em duas das estratégias mencionadas com recorrência: "realizar compostagem" e "alimentar animais", é possível identificar um equívoco dos respondentes em relação ao que significa desperdiçar alimentos. Uma percepção similar foi encontrada por Wessolowski (2019). Apesar de ambas serem formas de lidar com os resíduos e reduzirem os impactos ambientais associados à sua disposição, ainda configuram desperdício, pois o alimento destinado ao consumo humano é desviado para outro fim (Wessolowski, 2019).

Além disso, essa percepção equivocada pode fazer com que os indivíduos percam a sensibilidade para o desperdício alimentar, pois acreditam que estão fazendo "boas ações" que anulam ou compensam o desperdício. O estudo conduzido por Neff et al., (2015) concluiu que $41 \%$ dos participantes que praticam compostagem relataram que, uma vez que realizam essa prática, o descarte de alimentos em suas residências não os incomodava. Porpino et al. (2015) relatam ainda 
que algumas famílias não percebem a ocorrência de desperdício, ou o justificam, quando destinam sobras de alimentos para alimentação animal.

Algumas respostas sugerem que os indivíduos acreditam precisar de orientações e novas informações para lidar com o desperdício mais eficientemente. Trechos que se referem a "mais informação", "divulgação", "buscar conhecimento" aparecem em diversas respostas e alguns participantes da pesquisa declararam ainda estar "procurando soluções" para o problema. Esse resultado corrobora com um dos destaques do Relatório de 2021 do Índice de Desperdício de Alimentos (UNEP, 2021), de que os cidadãos precisam de ajuda e orientação para reduzir o desperdício de alimentos em residências e, mais uma vez, valida a importância de estudos e iniciativas com esse escopo.

\section{CONSIDERAÇÕES FINAIS}

O desperdício de alimentos é um desafio global com múltiplas implicações sociais, ambientais e econômicas. Por esse motivo, o combate ao desperdício deve integrar esforços multidisciplinares. Iniciativas públicas e privadas podem contribuir para o desenvolvimento e propagação de estratégias para prevenção e redução do desperdício alimentar.

Esse estudo buscou identificar alguns dos principais fatores envolvidos no desperdício alimentar em residências no ERJ através da condução de uma pesquisa de opinião. $O$ estudo apontou resultados que permitem identificar quais os principais desafios enfrentados para à redução do desperdício, bem como as oportunidades para ação. Através do estudo, identificou-se que as principais causas para ocorrência do desperdício alimentar são comportamentais. Em geral, envolvem a falta de planejamento e o preparo em abundância. Um adequado planejamento da compra e preparo dos alimentos, bem como a correta conservação e armazenagem dos alimentos pode reduzir significativamente o desperdício. Apesar de um comportamento persistente ser difícil de ser corrigido, durante a pesquisa foi observado que, de forma geral, as pessoas sentem-se incomodadas com a prática do desperdício e têm interesse em mudar o seu comportamento para evitá-lo, precisando para isso de orientações práticas.

Os achados da pesquisa demonstram que as famílias com maior sensibilidade a questões ambientais tendem a desperdiçar menos. Por outro lado, foi constatada uma baixa associação do desperdício alimentar com impactos ambientais entre as famílias em comparação com a percepção de aspectos sociais e econômicos.

Dessa forma, evidencia-se a importância de que haja, antes de tudo, um fortalecimento da consciência ambiental dos indivíduos. Só então, a próxima medida poderá ser eficaz: reforçar a correlação desperdício alimentar-meio ambiente, através de abordagens mais diretas e específicas sobre os impactos ambientais associados ao desperdício. Assim, sensibilizados pelo entendimento da dimensão ambiental do desperdício, os indivíduos tenderiam a reduzi-lo.

$\mathrm{O}$ aproveitamento integral de alimentos apresenta-se como um grande aliado à redução do desperdício alimentar e à geração de resíduos orgânicos (Laurindo \& Ribeiro). Existem diversas iniciativas para divulgação de receitas à base de partes não convencionais dos alimentos. Ainda assim, o desconhecimento de receitas, foi apontado por 75,7 \% das famílias como principal limitação para a prática do aproveitamento integral dos alimentos. Portanto, a dificuldade de acesso das famílias a essas receitas, não se trata da carência de iniciativas a esse respeito, mas sugere um alcance ainda limitado das iniciativas existentes. $\mathrm{O}$ mesmo acontece referente às orientações para gestão adequada dos alimentos.

Ressalta-se que o estudo e os resultados encontrados representam uma contribuição para a pesquisa nacional no âmbito Desperdício alimentar. Como limitações desse estudo pode-se apontar a utilização de uma metodologia que não permitiu avaliar quantitativamente o desperdício. Além disso, os resultados de frequência de desperdício alimentar refletem apenas a auto avaliação dos respondentes. Como sugestão para trabalhos futuros indica-se a realização de estudos que analisem 
os impactos da pandemia de COVID-19 na ocorrência de desperdício alimentar, de forma que esse tema seja previsto em políticas de recuperação da pandemia.

Por fim, observa-se uma tendência recente de crescimento da abordagem da pauta desperdício alimentar ao nível mundial e no Brasil. O fortalecimento de discussões sobre o desperdício, integrado à prevenção de mudanças climáticas é um passo importante para o enfrentamento dessas questões globais, criando um cenário promissor de cooperação na busca de soluções para o grande desafio de atingir segurança alimentar, sustentabilidade ambiental e desenvolvimento socioeconômico e, consequentemente, para o cumprimento dos Objetivos de Desenvolvimento Sustentável.

\section{REFERÊNCIAS}

Aiolfi, A. H., \& Basso, C. (2013). Preparações elaboradas com aproveitamento integral dos alimentos. Disciplinarum Scientia| Saúde, 14(1), 109-114.

Assembleia Geral da ONU (2015). Transforming our world: the 2030 Agenda for Sustainable Development, A/RES/70/1, 21 October 2015.

Associação Brasileira das Empresas de Limpeza Pública (2019, 27 novembro). Os descaminhos do lixo. [Post da web] Recuperado de: http://abrelpe.org.br/brasil-produz-mais-lixo-mas-nao-avancaem-coleta-seletiva/.

Azevedo, P. B. de, Leite, J. C. A., Oliveira, W. S. N. de, Silva, F. M. da, \& Lima Ferreira, P. M da. (2015). Diagnóstico da degradação ambiental na área do lixão de Pombal, PB. Revista Verde de Agroecologia e Desenvolvimento Sustentável, 10(1), 20-34. doi: 10.18378/rvads.v10i1.3294.

Baptista, P., Campos, I., Pires, I., \& Vaz, S. J. (2012). Do campo ao garfo. Desperdício alimentar em Portugal. Lisboa: CESTRAS

Boaventura, L. T. A., Frades, L. P., Weber, M. L., \& Pinto, B. O. S. (2017). Conhecimento de manipuladores de alimentos sobre higiene pessoal e boas práticas na produção de alimentos. Revista Univap, 23(43), 53-62.

Borges, M. P., Souza, L. H. R., Pinho, S. D., \& Pinho, L. D. (2019). Impacto de uma campanha para redução de desperdício de alimentos em um restaurante universitário. Engenharia Sanitária $e$ Ambiental,24(4), 843-848. doi: 10.1590/s1413-41522019187411.

Bravi, L., Francioni, B., Murmura, F., \& Savelli, E. (2020). Factors affecting household food waste among young consumers and actions to prevent it. A comparison among UK, Spain and Italy. Resources, Conservation and Recycling, 153, 104586

Bressiani, J., Schwarz, K., Gatti, R. R., Demário, R. L., \& Freire, P. L. I. (2017). Desperdício alimentar X aproveitamento integral de alimentos: elaboração de bolo de casca de banana. Uniciências, 21(1), 39-44.

Comissão Europeia (2015). Plano de ação da EU para a economia circular. Disponível em http://ec.europa.eu/environment/circular-economy/index_en.htm 
Departamento Intersindical de Estatística e Estudos Socioeconômicos. Valor da cesta básica aumenta em praticamente todas as capitais em 2019. Nota à imprensa. São Paulo, 09 jan. 2020.Recuperado de: https://www.dieese.org.br/analisecestabasica/2019/201912cestabasica.pdf.

Dias, C., \& Verona, A. (2019). Excesso de peso, obesidade e educação no Brasil. Revista Saúde, 45(2), 1-08.

Empresa Brasileira de Pesquisa Agropecuária (2017, 16 outubro). Os desperdícios por trás do alimento que vai para o lixo. [Post da web] Recuperado de https://www.embrapa.br/en/hortalicas/busca-de-noticias/-/noticia/28827919/os-desperdicios-portras-do-alimento-que-vai-para-o-lixo.

Food and Agriculture Organization of the United Nations. (2015). Food Wastage Footprint and Climate Change. Roma: FAO.

Food and Agriculture Organization of the United Nations. (2013). Food wastage footprint: Impacts on natural resources - Summary report. Disponível em http://www.fao.org/docrep/018/ i3347e/i3347e.pdf.

Food and Agriculture Organization of the United Nations (2018,12 outubro). Graziano da Silva fala de metodologias contra perdas e desperdícios em Fórum na UniCamp. [Post da web] Recuperado de http://www.fao.org/brasil/noticias/detail-events/pt/c/1157134/

Food and Agriculture Organization of the United Nations (Produtora). (2016) Food wastage footprint. Disponível em https://www.youtube.com/watch?v=IoCVrkcaH6Q

FAO, IFAD, UNICEF, WFP and WHO. 2020. The State of Food Security and Nutrition in the World 2020. Transforming food systems for affordable healthy diets. Rome, FAO.

Guimarães, P. R. B. (2008). Métodos quantitativos estatísticos.

Hall, K. D., Guo, J., Dore, M., \& Chow, C. C. (2009). The progressive increase of food waste in America and its environmental impact. PloS one, 4(11), e7940. https://doi.org/10.1371/journal.pone.0007940

Heck, M., Pilcher, J., Ray, K., \& Brito, E. (2018). Quando comer se torna um negócio. Revista de Administração de Empresas, 58(3), 217-222. doi: 10.1590/s0034-759020180302

Hegnsholt, E., Unnikrishnan, S., Pollmann-Larsen, M., Askelsdottir, B., \& Gerard, M. (2018). Tackling the 1.6-billion-ton food loss and waste crisis. The Boston Consulting Group.

Hoekstra, A. Y., Chapagain, A. K., Aladaya, M. M., \& Mekonnen, M. M. (2011). Manual de avaliação da pegada hídrica: estabelecendo o padrão global. São Paulo: Instituto de Conservação Ambiental.

Instituto Brasileiro de Geografia e Estatística. (2019). Divisão regional, segundo as mesorregiões, microrregiões geográficas e municípios Estado do Rio de Janeiro - 2019.

Laurindo, T. R., \& Ribeiro, K. A. R. (2014). Aproveitamento integral de alimentos. Interciência \& Sociedade, $3(2)$. Recuperado
http://revista.francomontoro.com.br/intercienciaesociedade/article/view/57 
Leal, A. Análise da disposição final dos resíduos sólidos urbanos no Distrito de Pilar-Ba. Revista ComSertões, v. 4, n. 1, 2016.

Martindale, W. (2017). The potential of food preservation to reduce food waste. Proceedings of the Nutrition Society, 76(1), 28-33. doi: 10.1017/S0029665116000604

Masson-Delmotte, V., Zhai, P., Pörtner, H. O., Roberts, D., Skea, J., Shukla, P. R., ... \& Connors, S. (2018). Global warming of 1.5 C An IPCC Special Report. Report of the Intergovernmental Panel on Climate Change, 265.

Mbow, C. et al., (2019). Food Security. In: Climate Change and Land: An IPCC Special Report on Climate Change, Desertification, Land Degradation, Sustainable Land Management, Food Security, and Greenhouse Gas Fluxes in Terrestrial Ecosystems. Geneva: Intergovernmental Panel on Climate Change (IPCC).

Nahman, A., Lange, W. de, Oelofse, S., \& Godfrey, L. (2012). The costs of household food waste in South Africa. Waste Management, 32(11), 2147-2153. doi: 10.1016/j.wasman.2012.04.012.

Neff, R. A., Spiker, M. L., \& Truant, P. L. (2015). Wasted food: US consumers' reported awareness, attitudes, and behaviors. PloS one, 10(6), e0127881. doi: 10.1371/journal.pone.0127881

Nespolo, C. R., de Oliveira, F. A., Pinto, F. S. T., \& Olivera, F. C. (2015). Práticas em tecnologia de alimentos. Artmed Editora.

ONU News (2013, 21 outubro). Reducing food loss, waste key to fighting hunger, UN official stresses at global fórum [Post da web]. Recuperado de https://news.un.org/en/story/2013/10/453382\#.UmfQv_lwroO.

Paiva Júnior, F. G., de Souza Leão, A. L. M., \& de Mello, S. C. B. (2011). Validade e confiabilidade na pesquisa qualitativa em administração. Revista de Ciências da Administração, 13(31), 190-209. doi: 10.5007/2175-8077.2011v13n31p190.

Porpino, G., Lourenço, C. E., Araújo, C. M., \& Bastos, A. (2018). Intercâmbio Brasil-União Europeia sobre desperdício de alimentos. Relatório final de pesquisa, Diálogos Setoriais União Europeia-Brasil. Recuperado de www. sectordialogues. org/publicacao.

Porpino, G., Parente, J., \& Wansink, B. (2015). Food waste paradox: antecedents of food disposal in low income households. International journal of consumer studies, 39(6), 619-629. doi: 10.1111/ijcs. 12207

Quested, T., \& Murphy, L. (2014). Household food and drink waste: a product focus. United Kingdom: Banbury (UK): Waste and Resources Action Programme.

Rio de Janeiro. Governo do estado. I Plano Estadual de Segurança Alimentar e Nutricional Sustentável do Estado do Rio de Janeiro (MINUTA para Consulta Pública). 2018. Recuperado de: http://rj.gov.br/MinutaPLESANS.pdf.

Searchinger, T, Waite, R, Hanson, C, Ranganathan, J, Dumas, P, Matthews, E \& Klirs, C. (2019). Creating a sustainable food future: A menu of solutions to feed nearly 10 billion people by 2050 . Final report, Month: July . World Resources Institute, 1-564. 
Shukla, P. R., Skea, J., Calvo Buendia, E., Masson-Delmotte, V., Pörtner, H. O., Roberts, D. C., ... \& Ferrat, M. IPCC (2019). Climate Change and Land: an IPCC special report on climate change, desertification, land degradation, sustainable land management, food security, and greenhouse gas fluxes in terrestrial ecosystems.

Stangherlin I. C. \& de Barcellos M. D. (2018). Desperdício de alimentos: uma análise de diversas variáveis de influência que afetam o consumidor. In Zaro, M. (Ed), Desperdício de alimentos: velhos hábitos, novos desafios. (pp.114-133) Caxias do Sul, RS: Editora da Universidade de Caxias do Sul-Educs.

Storck, C. R., Nunes, G. L., de Oliveira, B. B., \& Basso, C. (2013). Folhas, talos, cascas e sementes de vegetais: composição nutricional, aproveitamento na alimentação e análise sensorial de preparações. Ciência Rural, 43(3), 537-543. Recuperado de

https://www.redalyc.org/articulo.oa?id=331/33125632027

Tortora, G. J., Case, C. L., \& Funke, B. R. (2016). Microbiologia (12 ed.). Porto Alegre. RS: Artmed Editora.

United Nations Environment Programme (2021). Food Waste Index Report 2021. Nairobi.

Vasques, C. T., \& Madrona, G. S. (2016). Aplicação de checklist para avaliação da implantação das boas práticas em uma unidade de alimentação e nutrição. Higiene Alimentar, 30(252/253), 54-58.

van Geffen, E. J., van Herpen, H. W. I., \& van Trijp, J. C. M. (2017). Quantified consumer insights on food waste: Pan-European research for quantified consumer food waste understanding: D1. 4. Refresh.

Vasconcelos, M. A. D. S., \& Melo, F. A. B. D. (2010). Conservação de alimentos.

Viana, K. L. S., \& de Souza, A. L. M. (2016). Avaliação do índice de resto ingestão, antes e durante uma campanha educativa, em unidade de alimentação e nutrição (UAN), Porto VelhoRO. Connection Line, (14). doi: 10.18312

Wessolowski, L. O. (2019). Proposição de diretrizes para minimizar o desperdício de alimentos em unidades familiares da base da pirâmide. (Dissertação de mestrado). Recuperado de http://repositorio.jesuita.org.br/handle/UNISINOS/8664

Wunderlich, S. M., \& Martinez, N. M. (2018). Conserving natural resources through food loss reduction: Production and consumption stages of the food supply chain. International Soil and Water Conservation Research, 6(4), 331-339.

Zago, V. C. P., \& Barros, R. T. D. V. (2019). Gestão dos resíduos sólidos orgânicos urbanos no Brasil: do ordenamento jurídico à realidade. Engenharia Sanitária e Ambiental, 24(2), 219-228. doi: 10.1590/s1413-41522019181376

\section{Como citar:}

Aragão, L. de O., Elabras-Veiga, L. B., \& de Souza, S. L. Q. (2021). Desperdício Alimentar em Residências no Estado do Rio de Janeiro: Alternativas para Redução. Revista De Gestão Social E Ambiental, 15, e02739. https://doi.org/10.24857/rgsa.v15i2.2739 